Check for updates

Cite this: Soft Matter, 2017, 13,3042

Received 25th October 2016, Accepted 24th March 2017

DOI: $10.1039 / c 6 s m 02412 b$

rsc.li/soft-matter-journal

\title{
Front microrheology of the non-Newtonian behaviour of blood: scaling theory of erythrocyte aggregation by aging
}

\author{
C. Trejo-Soto, ${ }^{a b}$ E. Costa-Miracle, (DD ${ }^{\text {bcd }}$ I. Rodriguez-Villarreal, ${ }^{b}$ J. Cid, ${ }^{e}$ M. Castro, ${ }^{f}$ \\ T. Alarcon ${ }^{\text {bcdg }}$ and A. Hernandez-Machado (iD) *abdh
}

\begin{abstract}
We introduce a new framework to study the non-Newtonian behaviour of fluids at the microscale based on the analysis of front advancement. We apply this methodology to study the non-linear rheology of blood in microchannels. We carry out experiments in which the non-linear viscosity of blood samples is quantified at different haematocrits and ages. Under these conditions, blood exhibits a power-law dependence on the shear rate. In order to analyse our experimental data, we put forward a scaling theory which allows us to define an adhesion scaling number. This theory yields a scaling behaviour of the viscosity expressed as a function of the adhesion capillary number. By applying this scaling theory to samples of different ages, we are able to quantify how the characteristic adhesion energy varies as time progresses. This connection between microscopic and mesoscopic properties allows us to estimate quantitatively the change in the cell-cell adhesion energies as the sample ages.
\end{abstract}

\section{Introduction}

The non-linear microrheology of several types of complex fluids has attracted a great deal of attention in recent times, ${ }^{1-3}$ particularly regarding the properties of a number of complex biological fluids. ${ }^{4-9}$ In spite of a wealth of studies regarding the physical characterisation of blood, ${ }^{10-13}$ the characterisation of its microrheological properties is a much more open field of study. The non-linear rheological properties of blood are mainly determined by the presence of red blood cells (RBCs) suspended in plasma. ${ }^{14-17}$ Some haematological pathologies have been associated with blood rheological properties such as viscosity, as for example the hyperviscosity syndrome, which is a set of symptoms triggered by an increase in blood viscosity, ${ }^{18}$

\footnotetext{
${ }^{a}$ Departament de Física de la Matèria Condensada, Facultat de Física, Universitat de Barcelona, Diagonal 645, E-08028 Barcelona, Spain. E-mail: a.hernandezmachado@gmail.com

${ }^{b}$ Centre de Recerca Matemàtica, Edifici C, Campus de Bellaterra, 08193 Bellaterra, Barcelona, Spain

${ }^{c}$ Departament de Matemàtiques, Universitat Autònoma de Barcelona, 08193 Bellaterra, Barcelona, Spain

${ }^{d}$ Barcelona Graduate School of Mathematics (BGSMath), Barcelona, Spain

${ }^{e}$ Servicio de Hemoterapia y Hemostasia, Hospital Clinic de Barcelona, Barcelona, Spain

${ }^{f}$ GISC and Grupo de Dinámica No Lineal (DNL), Escuela Técnica Superior de Ingeniería (ICAI), Universidad Pontificia Comillas, E-28015 Madrid, Spain

${ }^{g}$ ICREA, Pg. Llus Companys 23, 08010 Barcelona, Spain

${ }^{h}$ Institute of Nanoscience and Nanotechnology (IN2UB), Universitat de Barcelona, Barcelona, Spain
}

resulting in vascular occlusion, ${ }^{19}$ particularly in the microcirculation. These symptoms are observed in sickle cell anemia due to loss of deformability of red blood cells. ${ }^{20}$ It has been observed that severe malaria also produces the symptoms associated with hyperviscosity syndrome. ${ }^{21,22}$ Based on this concept, new avenues have opened for the diagnosis of haematological pathologies based on the analysis of the microrheological properties of blood.

Advances in experimental approaches to the rheological properties of blood are starting to be complemented by quantitative modelling, both theoretical and computational. A number of studies have focused on the role of red blood cell (RBC) elasticity. ${ }^{23-30}$ Further to the role of RBC elasticity, Fedosov et al. ${ }^{14}$ analysed the role of RBC aggregation in blood rheology which has emerged as a fundamental ingredient to understand the non-Newtonian behaviour of blood. In this paper, we present the experimental results and develop a theoretical framework which allows us to further explore the role of RBC aggregation in the non-linear rheology of blood. We have carried out experiments at the microscale using a device aimed to track the dynamics of blood in microchannels. Working at that scale our setup has two main advantages. First, micro-fabrication allows for improved control of the fluid-flow conditions at a very low cost and using microliter-sized blood samples. ${ }^{31-33}$ Second, our microfluidic device allows us to directly track the advancement of the air-blood interface using an optical microscope.

Our main contribution is to develop a scaling theory of the non-linear viscosity which allows us to account for the effects of 
aging on the aggregation of RBCs. To this end, we define an adhesion scaling number and obtain a scaling behaviour whereby the viscosity exhibits a power-law dependence on the capillary number. The adhesion number represents how the characteristic energy of aggregation between RBCs varies as age progresses. This framework provides a new methodology to characterise the rheology of non-Newtonian fluids based on the dynamics of the fluid-air interface. We dub this new concept front microrheology. In order to achieve this, we characterise a hydrodynamic regime in which the fluid-air interface moves at constant velocity. This regime is different from the usual approach (Washburn regime) in which the front velocity is a decreasing function of time. The analysis presented in this paper is a generalisation to non-Newtonian fluids of the one presented in ref. 34 . In particular, we define a new quantity associated with this nonWashburn regime, the front shear rate, which allows us to characterise the non-linear rheology of non-Newtonian fluids.

\section{Experimental set-up and measurements}

Our experimental setup (see Fig. 1) is designed such that we can characterise the non-linear rheology of blood through the advancement of the blood-air interface. The position of the advancing interface, $h(t)$, is recorded by means of an inverted microscope Optika XDS- 3 with an objective of magnitude $4 \times$. The images are recorded using a high speed camera Photron Fastcam Viewer 3 with a capture frame rate between 60 and 125 frames per second. The position uncertainty is determined by the size of the pixel. In our case, it is $\delta h=7.2 \mu \mathrm{m}$ over a $5 \mathrm{~mm}$ measured distance. A constant pressure drop is imposed by using a container located at a height $H$ with respect to the position of the microchannel. This container is connected to the microsystem by a microtube of a biocompatible material with a uniform internal circular cross-section of radius $r=0.127 \mathrm{~mm}$ and length $l_{\mathrm{t}}=0.430 \mathrm{~m}$. The microsystem is a rectangular microchannel of height $b=350 \mu \mathrm{m}$, width $w=1 \mathrm{~mm}$, and length $l_{\mathrm{c}}=4 \mathrm{~cm}$. The cross-section of our tube and the hydraulic diameter of the microchannel, $D_{\mathrm{h}}=0.52 \pm 0.02 \mathrm{~mm}$,

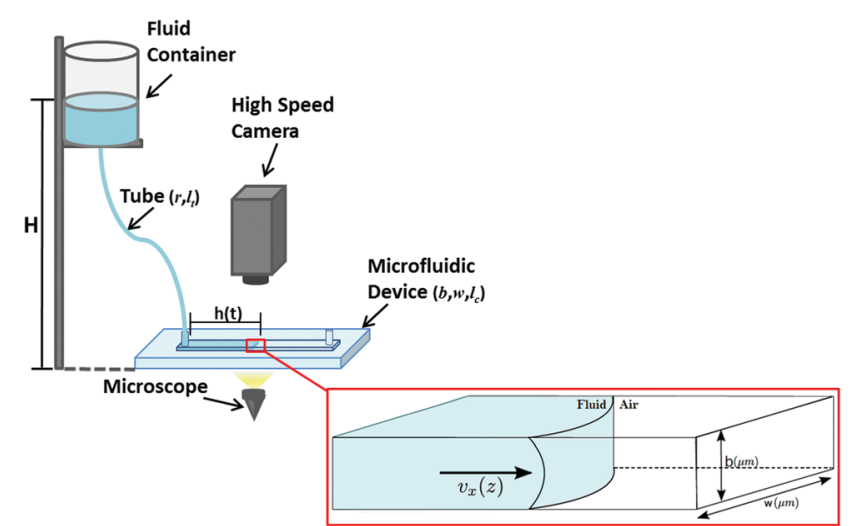

Fig. 1 Schematic representation of our experimental setup. An open reservoir at height $H$ pumps fluid into the microchannel through a tube of radius $r$ and length $l_{\mathrm{t}}$. are both typical of large arterioles and venules. The typical magnitude of the flow rate, $Q$, in our experiments is $Q=6 \mu \mathrm{min}^{-1}$. This flow rate is of the same order of magnitude as the one measured in capillaries. ${ }^{35}$ Within this experimental set-up, the wall shear rate of the tube, defined as $\dot{\gamma}=\frac{4 Q}{\pi r^{3}}$, varies within the range from 4.69 to $131.90 \mathrm{~s}^{-1}$. The microchannel is molded in a biocompatible hydrophobic silicone, PDMS (polydimethylsiloxane), on a glass substrate according to replica moulding and soft lithography microfabrication methods. ${ }^{34,36}$ The bottom surface of the microchannel is made of glass and the top and lateral surfaces are made of PDMS. In our experiments, we use human blood of varying ages (defined as the time elapsed since extraction) and haematocrits (the volume fraction occupied by the RBCs).

Blood samples were obtained from anonymous donors randomly selected. Blood samples for our experiments were delivered by the blood bank of the Hematology Department of the Clinical Hospital of Barcelona, in 10 or $5 \mathrm{ml}$ tubes with a heparin based anticoagulant. The use of these samples was authorised by the Bioethics Committee of the University of Barcelona. In order to preserve the state of the samples they were stored in a refrigerator at $4{ }^{\circ} \mathrm{C}$. Before any intervention, the samples were placed under an extraction hood to acquire room temperature, between $21 \pm 1{ }^{\circ} \mathrm{C}$. For each blood sample, we performed 2 different measurements, on each occasion we carried out 5 repetitions at each different pressure value.

The results obtained using the setup shown in Fig. 1 are summarised in Fig. 2, where we show how the front velocity, $\dot{h}$, varies as the effective pressure changes. The effective pressure is defined as $\rho g H-P_{\mathrm{L}}$, where $\rho$ is the blood density and $P_{\mathrm{L}}$ is the capillary pressure. We have calculated the capillary pressure based on the experimental determination of the contact angle, $\theta$, from the images of the advancing front using the well-known formula:

$$
P_{\mathrm{L}}=\gamma \cos \theta\left(\frac{1}{w}+\frac{1}{b}\right)
$$

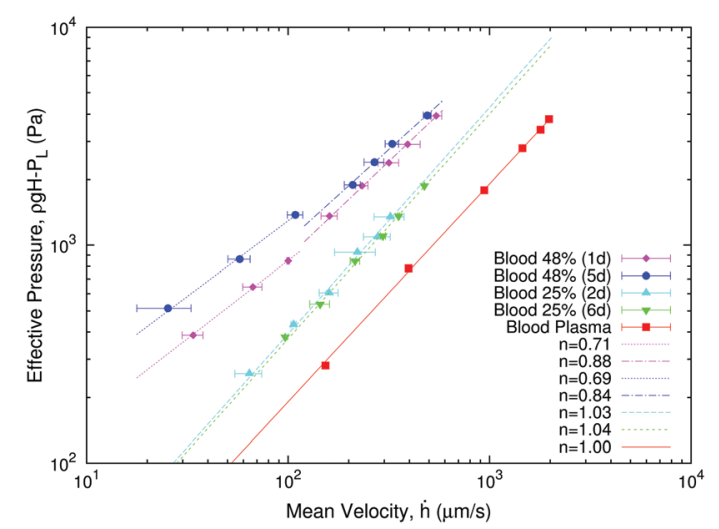

Fig. 2 Plot showing the dependence of the pressure on the mean front velocity for blood at different haematocrit values (48\% and $25 \%$ ) and for different post-extraction times. We observe that, in the case of whole blood (48\% haematocrit), two regimes are present: $n \simeq 0.70$ for small velocities (dotted lines) and $n \simeq 1$ (dashed line) for larger velocities. Blood at $25 \%$ haematocrit is observed to be Newtonian in the whole range of measured velocities, regardless of the age of the sample ( 2 or 6 days). For comparison, we add the results for plasma. 
Table 1 Viscosity parameters from eqn (10) for different blood sample haematocrits and ages. The low (high) shear rate regimes are defined in terms of the associated low (high) front velocity regimes in Fig. 2. It is worth mentioning that the viscosity obtained for blood plasma (last row in Table 1 ) is in the range $1.10-1.30 \mathrm{mPa}$ s at $37^{\circ} \mathrm{C}$ and independent of age and gender as measured using macroscopic viscosimeters ${ }^{38}$

\begin{tabular}{lll}
\hline Haematocrit & Exponent 1 $(n-1)$ & Prefactor $m$ \\
\hline $48 \%$ 1d (low shear rate) & -0.29 & 0.01190 \\
$48 \%$ 5d (low shear rate) & -0.31 & 0.02030 \\
$48 \%$ 1d (high shear rate) & -0.12 & 0.00830 \\
$48 \%$ 5d (high shear rate) & -0.16 & 0.01096 \\
$38 \%$ 1d & -0.07 & 0.00315 \\
$38 \%$ 5d & -0.12 & 0.00407 \\
25\% 2d & -0.004 & 0.00265 \\
25\% 6d & -0.01 & 0.00256 \\
Plasma & 0 & 0.00152
\end{tabular}

where $\gamma$ is the surface tension: $\gamma=0.061 \mathrm{~N} \mathrm{~m}^{-1}$. The contact angle has been found to vary between $\theta=64$ and $\theta=77$ degrees. Based on these experimental results, the range for the capillary pressure $P_{\mathrm{L}}$ is between 166 and $195 \mathrm{~Pa}$. The error associated with the hydrostatic pressure is given as a function of the uncertainty of the height of the fluid column, $\delta H=0.001 \mathrm{~mm}$. Considering the hydrostatic pressure, $P=\rho g H$, where the blood density, $\rho$, has been taken as $1050 \mathrm{~kg} \mathrm{~m}^{-3}$, the relative error of pressure ranges from $\delta P=15.16 \mathrm{~Pa}$ to $\delta P=49.39 \mathrm{~Pa}$.

As shown in Fig. 2, the effective pressure exhibits a power-law dependence on $\dot{h}$, with the precise slope and location of the curve being dependent on the haematocrit and the age of the sample. Interestingly, for blood at $48 \%$ haematocrit, regardless of the age of the sample, our experiments display two regimes: one at low velocity with $n \simeq 0.70$, and another at higher velocity with $n \simeq 1$, in the same fashion as plasma alone (see Table 1). By contrast, blood with a reduced haematocrit (25\%) has $n \simeq 1$ in the whole range and regardless of the age of the sample. This suggests that aging effects may be strongly dependent on the haematocrit.

\section{Mathematical model}

In order to rationalise the set of experiments presented above, we need to formulate a model which allows us to derive the expression of viscosity as a function of the setup parameters. In particular, we consider non-Newtonian fluids with power-law behaviour, i.e.

$$
\eta(\dot{\gamma}(z))=m(\dot{\gamma}(z))^{n-1}
$$

where $\dot{\gamma}(z)=\frac{\partial v_{x}}{\partial z}$ is the shear rate, $n$ is a constant which depends on the fluid and $m$ depends on $n$. In the special case $n=1$, the fluid is Newtonian and $m$ is, directly, the viscosity of the fluid (so, only in that case, it has units of Pa s). Although power-law models are only accurate for intermediate shear rates, ${ }^{37}$ Fig. 2 shows that it may accurately capture our working regime.

Using eqn (2), we can use the Navier-Stokes equation to derive a non-Newtonian Darcy equation associated with the advancement of the fluid front:

$$
\frac{\mathrm{d}}{\mathrm{d} z}(\eta(\dot{\gamma}(z)) \dot{\gamma}(z))=\frac{\Delta P}{h(t)}
$$

where we have neglected the inertial and transient effects as we operate at very low Reynolds numbers, $\mathrm{Re}=\dot{h} D_{\mathrm{h}} \rho / \eta$, which we estimate from our experimental results to vary between $\operatorname{Re}=0.00109$ and $\operatorname{Re}=0.0632$, and we assume that the fluid adapts almost instantaneously to changes in the front location. In eqn (3), $\Delta P=\rho g H-\Delta P_{\mathrm{t}}-P_{\mathrm{L}}$, with $P_{\mathrm{L}}$ being the capillary pressure and $\Delta P_{\mathrm{t}}$ the pressure drop across the inlet tube (see Fig. 1). Integrating eqn (3) we obtain:

$$
\dot{\gamma}(z)=\left(\frac{\Delta P}{m h(t)} z\right)^{1 / n}
$$

By integrating this expression and imposing a non-slip boundary at $z= \pm b / 2$, we obtain $v_{x}(z)$. We further define the flow rate, $Q$, as:

$$
Q=w \int_{-b / 2}^{b / 2} v_{x}(z) \mathrm{d} z=2 w\left(\frac{\Delta P}{m h(t)}\right)^{1 / n}\left(\frac{b}{2}\right)^{\frac{1}{n}+2}\left(\frac{1}{\frac{1}{n}+2}\right)
$$

from which we can derive the average front velocity, $\dot{h}: Q=b w \dot{h}$. Exploiting the relation between $Q$ and $\dot{h}$, we derive an equation for the dependence of $\Delta P$ on $\dot{h}$ :

$$
\rho g H-\Delta P_{\mathrm{t}}-P_{\mathrm{L}}=\frac{2 m}{b}\left(\frac{2}{b}\left(2+\frac{1}{n}\right)\right)^{n} h \dot{h}^{n}
$$

Using the same procedure for the inlet tube in cylindrical coordinates, we obtain an explicit expression for $\Delta P_{\mathrm{t}}$ in terms of the average flow velocity within the tube, $v_{\mathrm{t}}$. Flow conservation across the whole tube-microchannel system, $b w \dot{h}=\pi r^{2} v_{\mathrm{t}}$, allows us to couple both fluid systems to obtain a global expression which relates the front velocity with the pressure drop:

$$
\dot{h}(t)^{n}=\frac{\left(\rho g H-P_{\mathrm{L}}\right)}{\left[\frac{2 l_{\mathrm{t}}\left(\frac{1}{n}+3\right)^{n}}{r^{1+n}}\left(\frac{b w}{\pi r^{2}}\right)^{n}+\frac{h(t)\left(2+\frac{1}{n}\right)^{n}}{\left(\frac{b}{2}\right)^{1+n}}\right]}
$$

Under the conditions of our experimental setup, the second term in the denominator of eqn (7) is much smaller than the first term at all $t$, i.e. the resistance in the microchannel is much smaller than in the tube. Note that our results are not independent of the microchannel since the validity of our constant velocity regime depends on the geometrical parameters of both the microchannel and the inlet microtube. If these assumptions hold, $\dot{h}$ is a constant and we obtain a relation between $\rho g H$ and $\dot{h}$ given by:

$$
\rho g H-P_{\mathrm{L}}=m \frac{2 l_{\mathrm{t}}\left(\frac{1}{n}+3\right)^{n}}{r^{1+n}}\left(\frac{b w}{\pi r^{2}}\right)^{n} \dot{h}^{n}
$$

eqn (8) allows us to define a rescaled pressure, $\sigma$, and the shear rate, $\dot{\gamma}_{\mathrm{F}}(n)$, associated with the advancement of the front:

$$
\begin{gathered}
\sigma=\frac{r}{2 l_{\mathrm{t}}}\left(\rho g H-P_{\mathrm{L}}\right), \\
\dot{\gamma}_{\mathrm{F}}(n)=\frac{b^{2} w}{\pi r^{3}}\left(3+\frac{1}{n}\right)\left(\frac{\dot{h}}{b}\right)
\end{gathered}
$$


The quantity $\dot{\gamma}_{\mathrm{F}}$, which we refer to as the front shear rate, is central to our analysis, as it allows a characterisation of the non-linear rheology in terms of the average front velocity, as opposed to the local definition of the shear rate, commonly used in other approaches. We can now define a viscosity associated with the dependence of the rescaled pressure, $\sigma$, on the shear rate: $\sigma=\eta\left(\dot{\gamma}_{\mathrm{F}}\right) \dot{\gamma}_{\mathrm{F}}=m \dot{\gamma}_{\mathrm{F}}^{n}$, where $\eta\left(\dot{\gamma}_{\mathrm{F}}\right)$ is given by:

$$
\eta\left(\dot{\gamma}_{\mathrm{F}}\right)=m \dot{\gamma}_{\mathrm{F}}^{n-1}
$$

\section{Results}

\subsection{Non-linear rheology}

Following eqn (7) we determine under which experimental conditions we can consider that the advancement of the fluid front occurs at constant velocity. Such conditions are given in terms of the geometry of our setup and we make sure that they are fulfilled during our experiments. Furthermore, we can measure the velocity of the front as a function of the pressure drop. We then simply change the pressure drop by changing the height of the reservoir, $H$.

In Fig. 3, we show how the blood viscosity varies as the shear rate, $\dot{\gamma}_{\mathrm{F}}(n)$, changes. These results are associated with the ones shown in Fig. 2. We observe that our experimental results fit within our power-law ansatz eqn (10). The values of the parameters $m$ and $n$ are given in Table 1 . Although our results for the variation of viscosity as the front shear rate changes extend over one order of magnitude only, we note that our set-up allows for an extension of this range by merely changing the gap of the microchannel, $b$, as shown in eqn (9). The values obtained for viscosity, within the studied range of front shear rate, are very similar to those obtained by Cockelet et al. ${ }^{10}$

\subsection{Scaling theory of aging effects}

We start our analysis by addressing the effects of aging on blood viscosity. Fig. 4(a) and (b) show that, as the sample ages, RBCs organise themselves into structures called rouleaux.

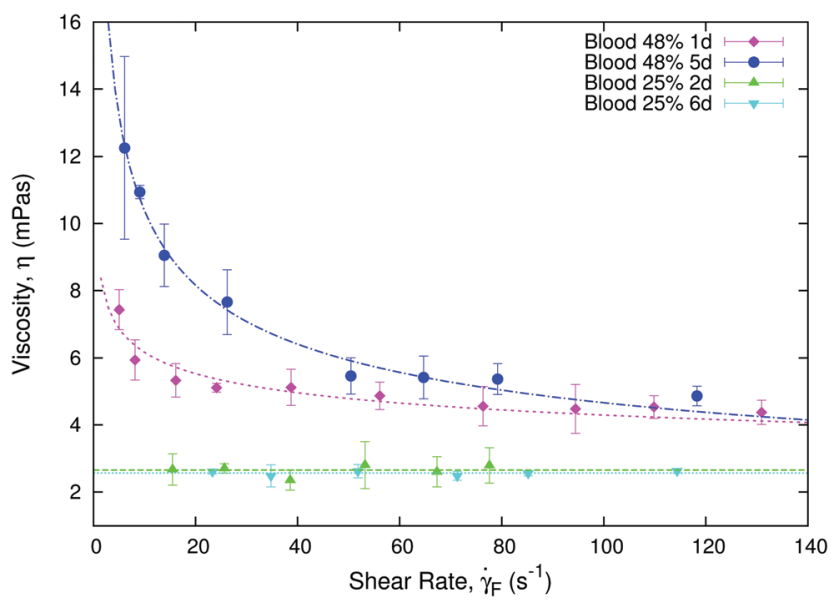

Fig. 3 Plot showing how the viscosity of blood varies as a function of the front shear rate, $\dot{\gamma}_{\mathrm{F}}$ for samples of different ages. We observe that as the sample ages, its viscosity increases. These results are associated with the experiments shown in Fig. 2. They have been obtained by using eqn (9) and (10).

Simulation results reported in ref. 14 have shown that the aggregation of RBCs into such structures affects the rheological properties of blood. Our experimental results (Fig. 4(a) and (b)) show that rouleaux appear due to aging of the samples, whereby we put forward that aging also affects blood viscosity.

To analyse the effects of aging, we fix the value of the haematocrit and study the behaviour of the blood sample as it ages. We introduce a new, non-linear scaling parameter, the adhesion scaling number, $A$, which quantifies the effects of aging on RBC aggregation. This quantity is defined as:

$$
A=\frac{\eta_{0} \dot{\gamma}_{\mathrm{F}}(n) d^{3}}{E\left(n, a, E_{0}\right)}
$$

where $\eta_{0}$ is the viscosity of plasma, $E$ is the energy scale associated with the aggregation energy between RBCs, ${ }^{14}$ which

(a)

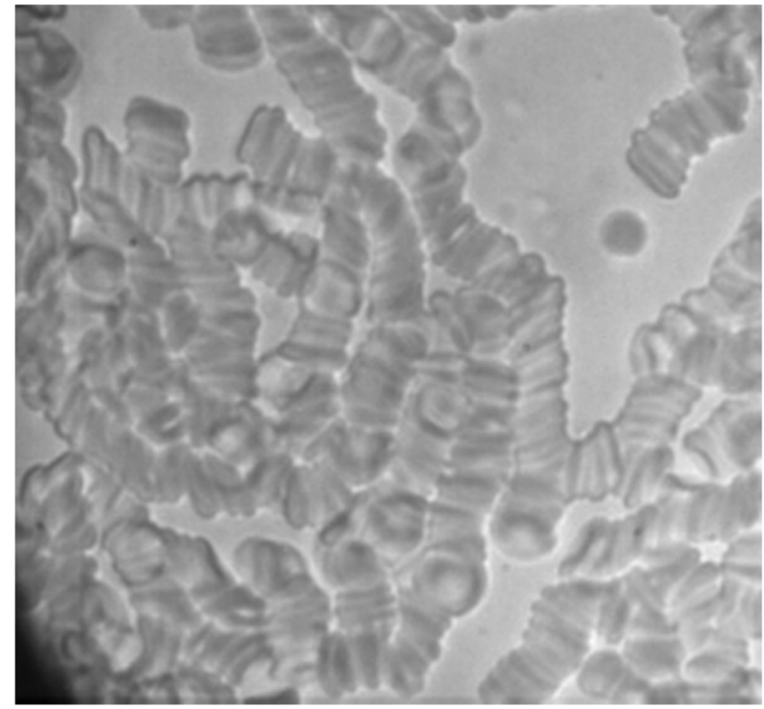

(b)

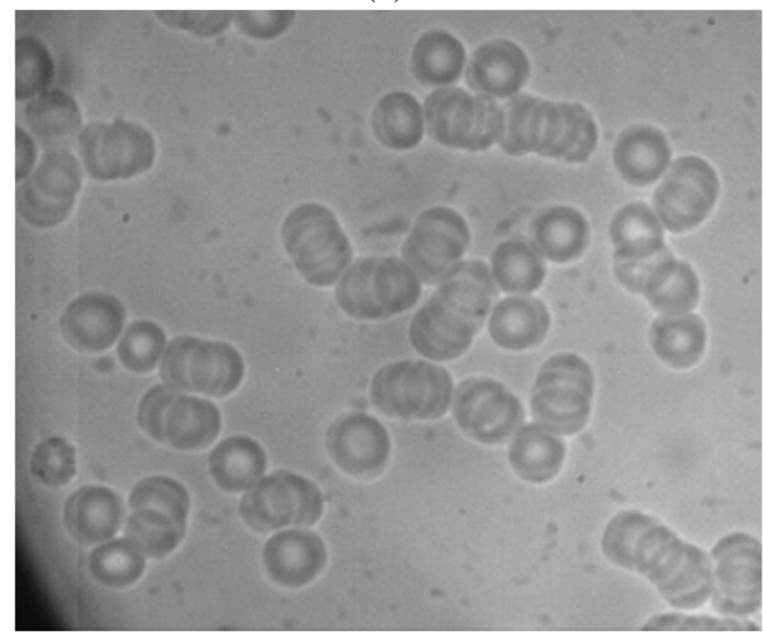

Fig. 4 (a) and (b) Photographs of the same sample at 5 days and 1 day obtained using a microscope OPTIKA XDS-3 with an objective of magnitude $100 \times$. We observe that, as the sample ages, erythrocytes aggregate into structures known as rouleaux. Our scaling theory addresses the effects of aging-induced aggregation on blood viscosity. 
depends on the exponent $n$, the age of the sample, $a$, and a reference value of RBC aggregation, $E_{0}$, which has been estimated to be of the order of $500 k_{\mathrm{B}} T .{ }^{14} \mathrm{~d}$ is the average diameter of a red blood cell. $A$ can be interpreted as the ratio between the characteristic viscous energy scale and the aggregation energy.

Our scaling ansatz claims that the viscosity, $\eta$, depends on the adhesion scaling number, $A$, alone:

$$
\frac{\eta}{\eta_{0}} \simeq \mu_{\mathrm{eff}}\left(H_{\mathrm{hem}}\right) A^{-\alpha}
$$

where $\mu_{\text {eff }}\left(H_{\text {hem }}\right)$ is a factor that accounts for the dependence of the normalised viscosity on the haematocrit, $H_{\text {hem }}$. Furthermore, our ansatz also states that $E\left(n, a, E_{0}\right)=k_{\mathrm{a}} E_{0}$, where the dimensionless scaling factor $k_{\mathrm{a}}$ is a function of the exponent $n$ and $a$. This scaling factor $k_{\mathrm{a}}$ accounts for the relative increase in the adhesion energy when the blood ages. Such an increase in the adhesion energy is reflected in the observation that rouleaux are more likely to form in older blood samples (see Fig. 4(a) and (b)) The scaling factor is obtained by collapsing

(a)

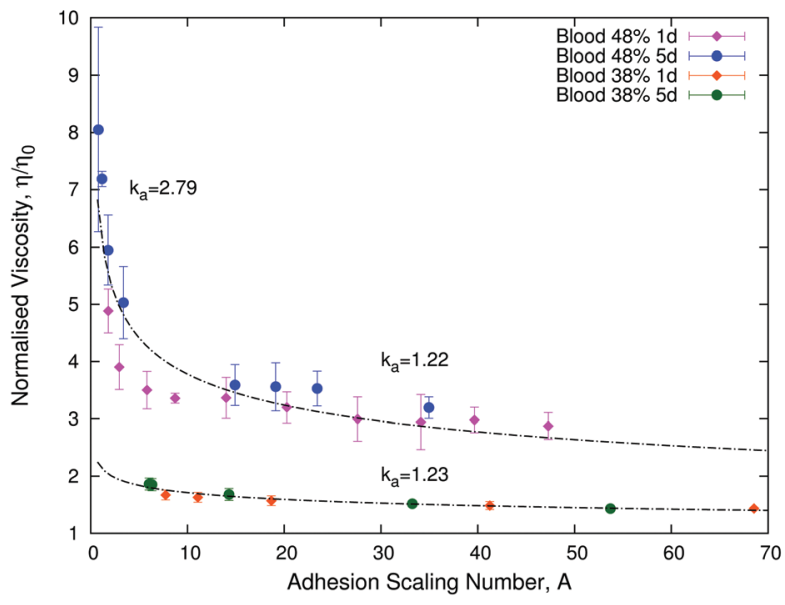

(b)

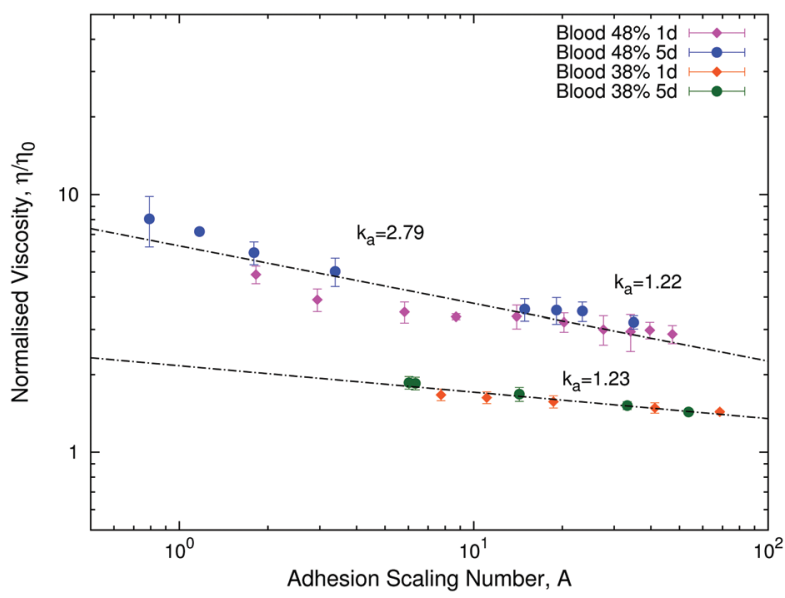

Fig. 5 Plots showing how the normalised viscosity, $\eta / \eta_{0}$, follows a universal curve as the adhesion scaling number (eqn (11)) changes. Panel (b) shows that this universal curve is well-described by a power-law in agreement with eqn (12). the curves for experimental data regarding the variation of the viscosity as the shear rate changes.

Fig. 5 shows the results for two different haematocrit values. These results show that our scaling ansatz can accurately account for the variation of viscosity with age. In particular, we observe that at lower values of the adhesion number, $A$, the effects of aging are more important than at higher values of $A$ (see Fig. 5, 48\% haematocrit sample). This behaviour is associated with the formation of rouleaux at low $A$ (see Fig. 4(a) and (b)). We have also analysed a different sample with a lower haematocrit (see Fig. 5, $38 \%$ haematocrit sample), for which we only studied the larger $A$ regime, in which we also find aging effects. The corresponding value of $k_{\mathrm{a}}$ is very similar in both samples for the same range of values of the adhesion number $A$, which shows that our assumption that $k_{\mathrm{a}}$ depends only on age and $n$ holds (see Table 1).

One could argue that the effects of varying the temperature could be important. We consider that changing the temperature would impact only the adhesion energy scale in eqn (10) and the viscosity of plasma, $\eta_{0}$. However, since the estimated adhesion energy scale is $500 k_{\mathrm{B}} T$, i.e. orders of magnitude higher than the thermal energy, we posit that the effects of temperature of RBC adhesion are likely to be negligible, and, therefore, the scaling behaviour described in this work should be robust to temperature changes. That is not to say that absolute values of viscosity will stay unchanged.

\section{Discussion and conclusions}

In this paper, we propose a new microrheological theory based on two fundamental points. First, we characterise a hydrodynamic regime in which the fluid-air interface moves at constant velocity. This regime is different from the usual approach in which the front velocity is a decreasing function of time. We then proceed to derive the appropriate physical description of the process within this regime. The former is achieved by using eqn (7). The usual setup, from which Washburn's regime is obtained, involves neglecting the first term in the denominator, thus yielding a system where the front moves at a time-dependent speed. By contrast, our set-up operates within the regime where the first term in the denominator is the dominant one. By neglecting the part inducing timedependence, we arrive at eqn (8) which allows us to define the new quantities associated with this particular regime: the front shear rate, $\dot{\gamma}_{\mathrm{F}}$, and the re-scaled pressure, $\sigma$. Finally, we define the viscosity as a function of the front shear rate as per eqn (10).

We have shown that variations in the non-linear viscosity of blood associated with aging can be explained by means of a scaling theory based on the introduction of an adhesion scaling number (eqn (11)). This quantity is defined as the ratio between the viscous energy and the aggregation energy. Our scaling theory allows us to quantify the increase in aggregation energy by aging with respect to its reference characteristic scale, $E_{0}$, by providing an estimation of the fold increase in $E_{0}, k_{\mathrm{a}}$. Note that further to its dependence on age, $k_{\mathrm{a}}$ is also a function of the shear rate. This reflects the fact that, at low shear rates, RBC aggregates are more likely to form. We further show that the viscosity exhibits a 
power-law behaviour over almost two orders of magnitude as a function of the scaling parameter $A$. We observe that blood exhibits a non-Newtonian behaviour at large haematocrits, which, at the shear rate scales of our experiments, is associated with aggregation of RBCs. These results allow us to better characterise the non-linear microrheological behaviour of blood.

The strength of our approach comes from the use of a front microrheometer that allows us to extract bulk non-linear viscosities from the simple observation of a moving air-fluid interface. This framework allows us to develop a non-linear microrheometer.

\section{Acknowledgements}

C. T.-S. and A. H.-M. gratefully acknowledge partial financial support from MINECO (Spain) under projects FIS2013-47949C01-01-P and FIS2016-78883-C2-1-P, and AGAUR project 2014SGR-878 and M. C. under projects FIS2013-47949-C01-02P and FIS2016-78883-C2-2-P. E. C.-M., I. R.-V., and T. A. gratefully acknowledge the Spanish Ministry for Science and Innovation (MICINN) for funding under grants MTM2015-71509-C2-1$\mathrm{R}$ and Generalitat de Catalunya for funding under grant 2014SGR1307. E. C.-M. and T. A. acknowledge support from the Ministry of Economy \& Competitivity (MINECO) for funding awarded to the Barcelona Graduate School of Mathematics under the "Maria de Maeztu" programme, grant number MDM-2014-0445. E. C.-M. acknowledges funding from MINECO through the scholarship BES-2015-072365. C. T.-S. acknowledges CONICYT the Chilean Ministry of Education for funding PhD Fellowship, Becas Chile.

\section{References}

1 T. M. Squires and T. G. Mason, Annu. Rev. Fluid Mech., 2010, 42, 413-438.

2 M. Aytouna, J. Paredes, N. Shahidzadeh-Bonn, S. Moulinet, C. Wagner, Y. Amarouchene, J. Eggers and D. Bonn, Phys. Rev. Lett., 2013, 110, 034501.

3 A. V. Ivlev, V. Steinberg, R. Kompaneets, H. Hofner, I. Sidorenko and G. E. Morfill, Phys. Rev. Lett., 2007, 98, 145003.

4 M. L. Gardel and D. A. Weitz, Microscale Diagnostic Techniques, Springer, Berlin, 2005.

5 D. Weihs, T. G. Mason and M. A. Teitell, Biophys. J., 2006, 91, 4296-4305.

6 D. Wirtz, Annu. Rev. Biophys., 2009, 38, 301-326.

7 A. Lindner, Phys. Fluids, 2014, 26, 101307.

8 J. Gachelin, G. Mino, H. Berthet, A. Lindner, A. Rousselet and E. Clement, Phys. Rev. Lett., 2013, 110, 268103.

9 T. G. Mason, K. Ganesan, J. H. van Zanten, D. Wirtz and S. C. Kuo, Phys. Rev. Lett., 1997, 79, 3282.

10 G. R. Cockelet, E. W. Merrill, E. R. Gilliland, H. Shin, A. Britten and R. E. Wells, Trans. Soc. Rheol., 1963, 7, 303-317.

11 S. Chien, S. Usami and J. F. Bertles, J. Clin. Invest., 1970, 49, 623-634.

12 A. R. Pries, D. Neuhaus and P. Gaehtgens, Am. J. Physiol.: Heart Circ. Physiol., 1992, 263, H1770-H1778.
13 G. B. Thurston and N. M. Henderson, Biorheology, 2006, 43, 729-746.

14 D. A. Fedosov, W. Pan, B. Caswell, G. Gompper and G. E. Karniadakis, Proc. Natl. Acad. Sci. U. S. A., 2011, 108, 11772-11777.

15 M. Brust, C. Schaefer, R. Doerr, L. Pan, M. Garcia, P. E. Arratia and C. Wagner, Phys. Rev. Lett., 2013, 110, 078305.

16 D. P. Monroe, J. Phys., 2013, 6, 18.

17 M. Thiebaud, Z. Shen, J. Harting and C. Misbah, Phys. Rev. Lett., 2014, 112, 238304.

18 H. Kwaan and A. Bongu, Semin. Thromb. Hemostasis, 1999, 25, 199-208.

19 J. M. Higgins, D. T. Eddington, L. Mahadevan and S. N. Bhatia, Proc. Natl. Acad. Sci. U. S. A., 2007, 104, 20496.

20 D. K. Wood, A. Soriano, L. Mahadevan, J. M. Higgins and S. N. Bhatia, Sci. Transl. Med., 2012, 4, 123 ra26.

21 V. Sitprija, M. Vongsthongsri, V. Poshyachinda and S. Arthachinta, Nephron, 1977, 18, 277-287.

22 J. P. Shelby, J. White, K. Ganesan and D. T. Chiu, Proc. Natl. Acad. Sci. U. S. A., 2007, 100, 14168.

23 Y. Park, C. A. Best, K. Badizadegan, R. R. Dasari, M. S. Feld, T. Kuriabova, M. L. H. A. J. Levine and G. Popescu, Proc. Natl. Acad. Sci. U. S. A., 2010, 107, 6731-6736.

24 Y. Park, C. A. Best, T. Auth, N. S. Gov, S. A. Safran, G. Popescu, S. Suresh and M. S. Feld, Proc. Natl. Acad. Sci. U. S. A., 2010, 107, 1289-1294.

25 B. Kaoui, G. Biros and C. Misbah, Phys. Rev. Lett., 2009, 103, 188101.

26 H. Noguchi and G. Gomper, Proc. Natl. Acad. Sci. U. S. A., 2005, 102, 14159-14164.

27 J. L. McWhirter, H. Noguchi and G. Gomper, Proc. Natl. Acad. Sci. U. S. A., 2009, 106, 6039-6043.

28 G. R. Lazaro, A. Hernandez-Machado and I. Pagonabarraga, Soft Matter, 2014, 10, 7195.

29 G. R. Lazaro, A. Hernandez-Machado and I. Pagonabarraga, Soft Matter, 2014, 10, 7207.

30 T. Savin, M. M. Bandi and L. Mahadevan, Soft Matter, 2016, 12, 562-573.

31 G. Whitesides, Nature, 2006, 442, 368-373.

32 P. Nghe, G. Degre, P. Tabeling and A. Ajdari, Appl. Phys. Lett., 2008, 93, 204102.

33 P. Nghe, S. M. Fielding, P. Tabeling and A. Ajdari, Phys. Rev. Lett., 2010, 104, 248303.

34 C. Trejo-Soto, E. Costa-Miracle, I. Rodriguez-Villarreal, J. Cid, T. Alarcon and A. Hernandez-Machado, PLoS One, 2016, 11, e0153559.

35 W. W. Nichols, M. F. ORourke and C. Vlachopoulos, McDonald's blood flow in arteries, Hodder Arnold, USA, 2011.

36 J. C. McDonald, D. C. Duffy, J. R. Anderson, D. T. Chiu, H. Wu, O. J. Schueller and G. M. Whitesides, Electrophoresis, 2000, 21, 27.

37 F. A. Morrison, Understanding rheology, Oxford University Press, USA, 2001.

38 G. Késmárky, P. Kenyeres, M. Rábai and K. Tóth, Clin. Hemorheol. Microcirc., 2008, 39, 243-246. 